Research Article

\title{
Optimal Sensor Placement for Inverse Finite Element Reconstruction of Three-Dimensional Frame Deformation
}

\author{
Yong Zhao $\mathbb{D}^{1},{ }^{1}$ Jingli Du $\mathbb{D}^{1},{ }^{1}$ Hong Bao $\mathbb{D}^{1},{ }^{1}$ and Qian $X \mathbf{u}^{2}$ \\ ${ }^{1}$ Key Laboratory of Electronic Equipment Structure Design, Ministry of Education, Xidian University, Xi'an 710071, China \\ ${ }^{2}$ Xinjiang Observatory, National Astronomical Observatories, Chinese Academy of Sciences, Urumqi 830011, China
}

Correspondence should be addressed to Hong Bao; hbao@xidian.edu.cn

Received 21 January 2018; Revised 19 May 2018; Accepted 5 June 2018; Published 4 July 2018

Academic Editor: Christopher J. Damaren

Copyright (c) 2018 Yong Zhao et al. This is an open access article distributed under the Creative Commons Attribution License, which permits unrestricted use, distribution, and reproduction in any medium, provided the original work is properly cited.

\begin{abstract}
The inverse finite element method (iFEM) for the 3D framework deformation reconstruction was introduced. As the process of iFEM did not require a priori knowledge, such as the modal shape, the loading, and the elastic-inertial material information of the structure, it presented high potential in the framework deformation reconstruction. With the current research, it was observed that the key step in the deformation reconstruction of the frame structure with iFEM was the section strains computing of the beam element from the surface strain measurements. The corresponding stability was severely affected by the placement of strain sensors. Therefore, it was necessary to discover a suitable sensor placement to maintain the stability of section strains computing. For this problem, one optimal model of sensor placement was proposed in this paper. Firstly, the well-separated eigenvalues were applied as the optimization target to construct the optimal model. Following, an optimal sensor placement was obtained through the optimal placement model solution, with the particle swarm optimization (PSO) method. Finally, the effectiveness of optimal placement was verified though the accuracy comparison of iFEM deformation reconstruction of a wing-like frame subjected to various loads for different schemes of sensor placement.
\end{abstract}

\section{Introduction}

Real-time reconstruction of structural deformation is a key technology in structural health monitoring (SHM) and control of mechatronics structure. For structures such as aircraft wings with embedded conformal antennas and high-sized frame structures that carry antennas, an accurate shape estimation is the prerequisite for the actuator adjustment to increase detection and communication quality [1-3]. The structural deformation reconstruction from in situ strain measurements commonly referred to as shape sensing presents high advantage in the deformation reconstruction of high-sized structures.

The key technology in shape sensing is to model the relationship between the in situ strain measurement and the displacement exactly. Certain authors modeled the relationship through methods such as neural and fuzzy nets $[4,5]$. Although these methods have good capabilities to model the relationship, the process of prediction model training requires high-sized training samples. Also, the mapping relation may be singular and ill conditioned when the training samples are insufficient. Other researchers treated the structural deformation as beam/plate bending and twist problems, through the linear regression prediction of a global or piecewise continuous base function, to model the relationship among the measured strain data and structural displacement.

In the literatures $[6,7]$, the global or piecewise continuous basis function methods were employed to fit the surface measured strain into a structure strain field. Consequently, the deformation of the structure was obtained through the strain-displacement relationship. This method was easy to implement, but its range of application and the accuracy of deformation estimation depended on the appropriate selection of the basis function and the weight coefficients. Modal shapes were used as a basis function in [8]. The displacements were reconstructed from measured strains with the modal transformation method. Adversely, the following 
disadvantages existed in this method: (1) the detailed material elasticity and inertial parameters were required to precisely construct the modal shapes; therefore, it was difficult to model the complex structure exactly. (2) The method required a computationally intensive eigenvalue analysis when applied to high-fidelity finite element models. On the basis of Euler-Bernoulli beam equation, Glaser et al. and Jute et al. determined the deflection of beam through the direct integration of discretely measured strains $[9,10]$. Particularly, Jute et al. applied the classical beam equation to develop a method to approximate the beam curvature through the discrete measured strain data integration [10]. The resultant one-dimensional solution displayed high accuracy in the deflection prediction, but this method failed to estimate the element deformation under multidimensional complex loads.

Shkarayev et al. developed a finite element-based methodology involving an inverse interpolation formulation that employed the surface measured strain to determine the loads and structural response of aerospace vehicles [11, 12]. By contrast, an appropriate quality function was required to select the most appropriate load case in this algorithm and this function was constructed based on computer simulations and experimental statistics.

Based on the Timoshenko theory and inverse finite element methodology (iFEM) framework proposed by Tessler and Spangler [13, 14], Gherlone et al. proposed the in situ measured strain data to reconstruct threedimensional displacement of the frame structures [15]. Due to the fact that only the displacement-strain relationship was used, this methodology could conduct deformation reconstruction without the prior knowledge of loads, materials, and inertial damping. Furthermore, these authors reproduced the structural deformation reconstruction of the single beam under loading case of the end-node force through iFEM [16]. The simulation and test results demonstrated that the scheme could accurately reconstruct the beam structure deformation under static load and force. Moreover, as stated in [16], the authors proposed four schemes of strain sensor placement and discovered that the locations where the strain sensors were placed would affect the deformation reconstruction accuracy, but the location selection for the sensor placement to retain the accuracy of reconstruction was not mentioned. Consequently, it was necessary to discover a common strategy to optimize the placement of strain sensors to guarantee the accuracy and stability of structural deformation reconstruction in iFEM.

Commonly, the optimal sensor placement schemes mainly included the modal kinetic energy (MKE) method, the effective independence (EI) method, the modal assurance criterion (MAC) matrix, and certain intelligent optimization algorithms based on the aforementioned methods $[17,18]$. Adversely, these placement schemes were only adapted for the deformed shape reconstruction of a structure. The relationship between the displacement field and the measured strain was derived from the structural kinematical equation, such as through finite element analysis method. For the iFEM, the relationship between the displacement and the measured strain was based on the mechanical property analysis of the structure. Consequently, the aforementioned optimal placement schemes did not apply to iFEM.

The key step in iFEM was the section strains computing from measured surface strain data. It was discovered that the locations where the strain sensors were placed would directly affect the section strains computing, as well as the accuracy and stability of deformation reconstruction. The section strains computing from measured strain data in iFEM could be regarded as parameter identification, while the identification processing could be regarded as the linear equation solution. Therefore, the differences between the eigenvalues of the transfer matrix were proposed, which was associated to the transfer relationship among section strains and measured surface strain data, to assess the distribution of strain sensors, as well as maximizing the difference as the optimal model target. Moreover, as the sensor locations contained three variates (a coordinate along the midaxis and two angles around the two axes), while every variate had its own range, the PSO method was proposed to solve the optimal model. Subsequently, two different schemes of sensor placement were compared to the optimal result obtained by the PSO, through ANSYS simulation. Finally, an aluminum wing-like structure model was subjected to different static loadings to verify the optimal placement scheme effectiveness. The reconstruction accuracies of all tests demonstrated that the optimal scheme was robust and accurate.

\section{Review of Beam Deformation Reconstruction through iFEM}

For the careful beam deformation reconstruction in threedimensional domain, the iFEM described the beam displacement field based on the Timoshenko beam theory and the corresponding section strains were deduced based on the small-strain hypothesis.

In the Timoshenko beam theory, the displacement field of an isotropic and straight beam with constant crosssection could be represented by Cartesian coordinates as follows $[15,16]$ :

$$
\begin{aligned}
& u_{x}(x, y, z)=u(x)+z \theta_{y}(x)-y \theta_{z}(x), \\
& v_{y}(x, y, z)=v(x)-z \theta_{x}(x), \\
& u_{z}(x, y, z)=w(x)+y \theta_{x}(x),
\end{aligned}
$$

where $u_{x}, v_{y}$, and $w_{z}$ are the point displacements along the $x$-, $y$-, and $z$-axes, respectively. $u(x), v(x)$, and $w(x)$ denote the displacements at $y=z=0 ; \theta_{x}(x), \theta_{y}(x)$, and $\theta_{z}(x)$ are the rotations regarding three coordinate axes (Figure 1). The six kinematic variables in one node along the middle axes can be grouped in a vector form as:

$$
\mathbf{u}=\left\{u, v, w, \theta_{x}, \theta_{y}, \theta_{z}\right\}^{T}
$$




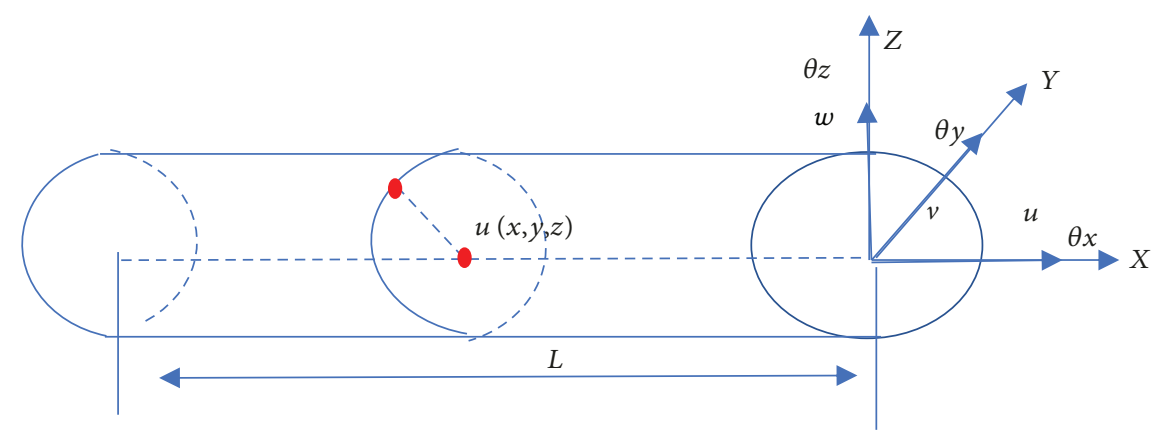

FIGURE 1: Beam geometry and kinematic variables.

Based on the small-strain hypothesis, the arbitrary section strain vector $\mathbf{e}(\mathbf{u})=\left[e_{1}, e_{2}, e_{3}, e_{4}, e_{5}, e_{6}\right]^{T}$ can be obtained from (1):

$$
\begin{aligned}
& e_{1}(x)=u_{x}(x) \\
& e_{2}(x)=\theta_{y, x}(x) \\
& e_{3}(x)=-\theta_{z, x}(x) \\
& e_{4}(x)=w_{x}(x)+\theta_{y}(x), \\
& e_{5}(x)=v_{x}(x)-\theta_{z}(x), \\
& e_{6}(x)=\theta_{x, x}(x)
\end{aligned}
$$

Following, the kinematic variable vector $\mathbf{u}$ could be confirmed when the section strain vector $\mathbf{e}(\mathbf{u})$ was obtained. In iFEM, the vector $\mathbf{e}(\mathbf{u})$ was replaced with the in situ section strain vector $\mathbf{e}^{\boldsymbol{\varepsilon}}$, computed from the measured surface strain data under the constraint of least square error.

$$
\boldsymbol{\varphi}(\mathbf{u})=\left\|\mathbf{e}(\mathbf{u})-\mathbf{e}^{\varepsilon}\right\|^{2} .
$$

Lastly, the relationship among the beam displacement and in situ strain measurements can be constructed as follows [15]:

$$
\mathbf{k}^{\mathrm{e}} \mathbf{u}^{\mathrm{e}}=\mathbf{f}^{\mathrm{e}}
$$

$\mathbf{k}^{\mathbf{e}}$ and $\mathbf{f}^{\mathbf{e}}$ were indicated as follows:

$$
\begin{aligned}
& \mathbf{k}^{\mathbf{e}}=\sum_{k=1}^{6} w_{k} k_{k}^{e}, k_{k}^{e}=\frac{L}{n} \sum_{i=1}^{n}\left[\mathbf{B}_{k}^{T}\left(x_{i}\right) \mathbf{B}_{k}\left(x_{i}\right)\right], \\
& \mathbf{f}^{\mathbf{e}}=\sum_{k=1}^{6} w_{k} f_{k}^{e}, \quad f_{k}^{e}=\frac{L}{n} \sum_{i=1}^{n}\left[\mathbf{B}_{k}^{T}\left(x_{i}\right) e_{k}^{\varepsilon}\left(x_{i}\right)\right],
\end{aligned}
$$

where $\mathbf{B}_{k}\left(x_{i}\right)$ is the coefficient matrix, derived from the element shape function. Once the location where the section strains were evaluated $\left(x_{i}\right)$ was determined, the coefficient matrix $\mathbf{B}_{k}\left(x_{i}\right)$ was fixed. $e_{k}^{\varepsilon}\left(x_{i}\right)$ are the in situ section strains computed from the measured surface strain data. $w_{k} k=(1,2, \ldots, 6)$ are the weighting coefficients used to reflect the effects of axial stretching, bending, twisting, and transverse shearing on the beam element. The corresponding expressions are as follows:

$$
\left\{w_{k}\right\}=\left\{\mathrm{w}_{1}^{0}, w_{2}^{0}\left(\frac{I_{y}^{e}}{A^{e}}\right), w_{3}^{0}\left(\frac{I_{z}^{e}}{A^{e}}\right), \mathrm{w}_{4}^{0}, \mathrm{w}_{5}^{0}, w_{6}^{0}\left(\frac{I_{p}^{e}}{A^{e}}\right)\right\},
$$

where $w_{k}^{0}(k=1,2, \ldots, 6)$ denote dimensionless weighting coefficients with initial values identically set as $1 ; A^{e}, I_{y}^{e}, I_{z}^{e}$, and $I_{p}^{e}$ are the cross-section area, second moments of the area according to the $y$ - and $z$-axes, and the polar moment of area of the beam element, respectively.

The key step in iFEM was the section strains computing $\mathbf{e}^{\varepsilon}=\left[e_{1}^{\varepsilon}\left(x_{i}\right), e_{2}^{\varepsilon}\left(x_{i}\right), \ldots, e_{6}^{\varepsilon}\left(x_{i}\right)\right]^{T}$ from the in situ surface strain measurements, $\varepsilon_{2}^{*}, i=1,2, \ldots, 6[15,16]$ :

$$
\begin{aligned}
\varepsilon_{2}^{*}\left(x_{i}, \theta_{i}, \beta_{i}\right)= & \varepsilon_{1}^{\varepsilon}\left(x_{i}\right)\left(c_{\beta_{i}}^{2}-v s_{\beta_{i}}^{2}\right)+e_{2}^{\varepsilon}\left(x_{i}\right)\left(c_{\beta_{i}}^{2}-v s_{\beta_{i}}^{2}\right) s_{\theta_{i}} R \\
& +e_{3}^{\varepsilon}\left(x_{i}\right)\left(c_{\beta_{i}}^{2}-v s_{\beta_{i}}^{2}\right) c_{\theta_{i}} R+e_{4}^{\varepsilon}\left(x_{i}\right) c_{\beta_{i}} s_{\beta_{i}} c_{\theta_{i}} \\
& -e_{5}^{\varepsilon}\left(x_{i}\right) c_{\beta_{i}} s_{\beta_{i}} s_{\theta_{i}}+e_{6}^{\varepsilon}\left(x_{i}\right) c_{\beta_{i}} s_{\beta_{i}} R
\end{aligned}
$$

with $\quad c_{\beta_{i}} \equiv \cos \beta_{i}, s_{\beta_{i}} \equiv \sin \beta_{i}, c_{\theta_{i}} \equiv \cos \theta_{i}, s_{\theta_{i}} \equiv \sin \theta_{i}$, where $\left(x_{i}, \theta_{i}, \beta_{i}\right)$ is the location, where the $i$ th strain sensor is set on the surface of the beam element, $v$ is the Poisson ratio, and $R$ is the external radius of the section (Figure 2).

Six different strain measurement data in one section were required to obtain section strains $\mathbf{e}^{\varepsilon}$ in one section, whereas the minimum of the sections where the section strains were evaluated differed for different loading cases. The minimum was 2 for the loading case of end-node forces and 3 for the loading case of uniformly distributed transverse forces [19]. This meant that the mininumber of sensors used to compute the section strains was 12 for end-node forces and 18 for uniformly distributed forces.

\section{Optimal Placement of Strain Sensors}

In this section, the number of strain sensors used to catch the beam surface strain and the equation computing the section strains in loading case of end-node force were described, firstly. Based on the latter equation section strains computing, the optimal placement of strain sensors was discussed. 


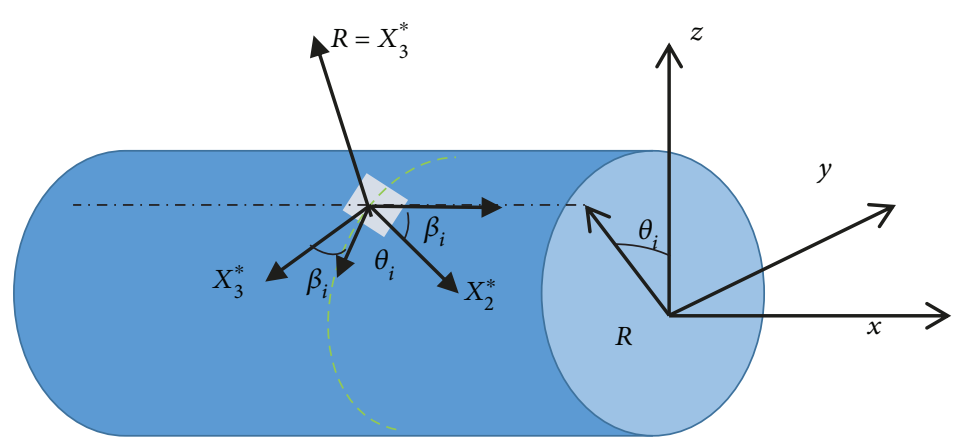

FIgURE 2: Location of strain gauge placed on beam element.

With the equilibrium equation analysis contribution, the section strains order on the cross-section along the beam element could be obtained. In the case of end-node forces, $e_{1}, e_{4}, e_{5}$, and $e_{6}$ were constants, while $e_{2}$ and $e_{3}$ were linear $[15,19]$. Following, the following equations to express section strains on arbitrary cross-section of the beam element through the above conclusion could be constructed as follows:

$$
\begin{aligned}
& e_{1}\left(x_{i}\right)=a_{1}, \\
& e_{2}\left(x_{i}\right)=a_{2} x_{i}+a_{3}, \\
& e_{3}\left(x_{i}\right)=a_{4} x_{i}+a_{5}, \\
& e_{4}\left(x_{i}\right)=a_{6}, \\
& e_{5}\left(x_{i}\right)=a_{7}, \\
& e_{6}\left(x_{i}\right)=a_{8} .
\end{aligned}
$$

The undecided parameters, $a_{1}, \ldots, a_{8}$, could be solved through the substitution of (9) into (8), with different surface strain measurements. Eight different strain measurements were required to determine the undecided parameters, which meant that each beam element required 8 sensors. Also, fixed proportion relations among $\mathrm{e}_{2}^{\prime}$ and $e_{4}$, as well as $e_{3}^{\prime}$ and $e_{5}$, existed:

$$
\begin{aligned}
& D_{y} e_{2}^{\prime}=G_{z} e_{4}, \\
& D_{z} e_{3}^{\prime}=G_{y} e_{5},
\end{aligned}
$$

with $G_{y} \equiv k_{y}^{2} G A, G_{z} \equiv k_{z}^{2} G A, D_{y} \equiv E I_{y}, D_{z} \equiv E I_{z}$, where $k_{y}^{2}$ and $k_{z}^{2}$ are the shear correction factors; $G$ and $E$ are the shear modulus and Young's modulus of the beam element, respectively. $A$ is the cross-section area of the beam element, while the second moments of area with respect to $y$ - and $z$-axes are $I_{y}$ and $I_{z}$, respectively. It was further noted that the ratios $D_{y} / G_{z}, D_{z} / G_{y}$ were constant once the external radius of the beam section was confirmed. Through (9), (10) could be modified as follows:

$$
\begin{aligned}
& e_{1}\left(x_{i}\right)=a_{1}, \\
& e_{2}\left(x_{i}\right)=a_{2} x_{i}+a_{3}, \\
& e_{3}\left(x_{i}\right)=a_{4} x_{i}+a_{5}, \\
& e_{4}\left(x_{i}\right)=\frac{D_{y}}{G_{z}} a_{2}, \\
& e_{5}\left(x_{i}\right)=\frac{D_{z}}{G_{y}} a_{4}, \\
& e_{6}\left(x_{i}\right)=a_{6} .
\end{aligned}
$$

Subsequently, the number of strain sensors used to solve the undecided parameters could be reduced to 6 . The latter process could lead to a solution of the following system of linear equations:

$$
\begin{aligned}
\varepsilon_{2}^{*}=T\left[\begin{array}{cccccc}
1 & 0 & 0 & 0 & 0 & 0 \\
0 & x_{i} & 1 & 0 & 0 & 0 \\
0 & 0 & 0 & x_{i} & 1 & 0 \\
0 & \frac{D_{y}}{G_{z}} & 0 & 0 & 0 & 0 \\
0 & 0 & 0 & \frac{D_{z}}{G_{y}} & 0 & 0 \\
0 & 0 & 0 & 0 & 0 & 1
\end{array}\right] *\left[a_{1}, \ldots, a_{6}\right]^{T} \\
= \\
\mathbf{Q}^{*}\left[a_{1}, \ldots, a_{6}\right]^{T}=\mathbf{Q}^{*} \mathbf{U A}\left[a_{1}, \ldots, a_{6}\right]^{T},
\end{aligned}
$$

with $\quad \varepsilon_{2}^{*}=\left[\varepsilon_{2}^{*}\left(x_{i}, \theta_{i}, \beta_{i}\right)\right]^{T}, T=\left[T_{1}, T_{2}, \ldots, T_{6}\right]^{T}, \quad \mathbf{U A}=$ $\left[a_{1}, \ldots, a_{6}\right]^{T}$, and $T_{i}=\left[c_{\beta}^{2}-v s_{\beta}^{2},\left(c_{\beta}^{2}-v s_{\beta}^{2}\right) s_{\theta} R,\left(c_{\beta}^{2}-v s_{\beta}^{2}\right) c_{\theta} R\right.$, $\left.c_{\beta} s_{\beta} c_{\theta}, c_{\beta} s_{\beta} s_{\theta}, c_{\beta} s_{\beta} R\right], i=1, \ldots, 6$.

Once the undecided parameters vector, UA was solved, the section strains along the beam element could be obtained from (11).

Nevertheless, through further research, it was discovered that the locations where the strain sensors were placed could impact the computing stability of the undecided parameters vector UA from the surface strain measurements through (12). The impertinent locations might even lead to the 
computing process becoming singular or ill conditioned, such as slight changes of the surface measured strain data might significantly change the section computing, which might result in the deformation reconstruction through iFEM becoming inaccurate or even to the reconstruction process failure. When the angles $\beta_{i}(i=1,2, \ldots, 6)$ were set to 0 , (12) was singular.

Taking into account that the main cause of ill-condition or singularity of the linear system of equations was that the differences among the eigenvalues of the coefficient matrix were too low, it was difficult to recognize the different system inputs. Kunsoo and Stein used the following conditions to define a well-conditioned matrix [20]:

(1) Minimized norm of the nonnormality matrix $\mathbf{M}$ in Schur form, as given by

$$
\mathbf{U}^{\mathrm{H}} \mathrm{FU}=\boldsymbol{\Lambda}+\mathbf{M},
$$

where $\mathbf{U}$ is a unitary matrix and $\mathbf{U}^{\mathbf{H}}$ is the conjugate transpose matrix of $\mathbf{U} ; \mathbf{F}$ is a normal matrix of $n \times n$ in size; $\boldsymbol{\Lambda}$ is a diagonal matrix with eigenvalues on diagonal elements; $\mathbf{M}$ is an upper-right off-diagonal matrix.

(2) Well-separated eigenvalues (such as large value in $\left.\min \left|\lambda_{i}-\lambda_{j}\right|, i \neq j\right)$.

In this paper, condition (2) was used to construct the optimal placement model of sensors in the loading case of end-node forces:

$$
\begin{aligned}
\text { find } f\left(\mathbf{Q}_{(\xi, \theta, \beta)}\right) & =\max \left(\min \left|\lambda_{i}-\lambda_{j}\right|\right), \quad i \neq j, i, j=1,2, \ldots, 6 \\
(\xi, \theta, \beta) & =\left[\xi_{1}, \theta_{1}, \beta_{1}, \ldots\right] \\
\text { s.t. } \quad \xi_{i} & \in[-0.8,0.8], \\
\theta_{i} & \in\left[-180^{\circ}, 180^{\circ}\right], \\
\beta_{i} & =0^{\circ} \text { or } 45^{\circ}, \\
i & =1,2, \ldots, 6
\end{aligned}
$$

where $\lambda_{i}, i=1,2, \ldots, 6$, are the eigenvalues of transformation matrix $\mathbf{Q}$. $(\xi, \theta, \beta)$ indicate the locations of the placed six sensors. $\xi_{i}=2 x_{i} / L-1 \in[-1,1]$ indicates a nondimensional axial coordinate with $x_{i} \in[0, L]$. In view of application environment in engineering, the locations on clamped and free end-nodes of the beam element are proven difficult to set sensors. Also, the beam surface curve would affect the accuracy of strain measurement. Therefore, $\xi_{i} \in[-0.8,0.8]$ was set, where only one sensor was placed at $\beta_{i}=45^{\circ}$ and the other five sensors were placed at $\beta_{i}=0^{\circ}$. The number of optimized sensor locations was 6 , and every location was determined by three parameters. Consequently, 18 parameters were required to be optimized. This was a multiparameter optimization, and the particle swarm optimization (PSO) algorithm was used to solve the optimal model, as the algorithm had good convergence speed and the optimal solution could be efficiently obtained through PSO in hyperspace.

Step 1. (initializing the PSO algorithm). The number of particles was set to $N=10$. The maximum iterations were $k_{\max }=200$, the number of iterations was initialized to $k=1$, and the threshold of the algorithm breaking was set to $M=$ 1000. The position of every particle was $a_{m}=$ $\left(\xi_{m 1}, \theta_{m 1}, \beta_{m 1}, \ldots, \xi_{m 6}, \theta_{m 6}, \beta_{m 6}\right)_{1 * 18}$, which was associated to the placed positions of strain sensors, while the corresponding velocity of every particle could be written as $v_{m}=$ $\left(v_{\xi 1}, v_{\theta 1}, v_{\beta 1}, \ldots, v_{\xi 6}, v_{\theta 6}, v_{\beta 6}\right)_{1 * 18}$.

The positions and velocities of the particles were initialized by discrete random values in the restrain range of the optimization model of (14), such as $\xi_{m j}, v_{\xi j} \in[-0.8,0.8], \theta_{m j}, v_{\theta j} \in$ $\left[-180^{\circ}, 150^{\circ}, \ldots, 180^{\circ}\right]$, and $\beta_{m j}, v_{\beta j}=0^{\circ}$ or $45^{\circ}(m=1, \ldots$, $50 ; j=1,2, \ldots, 6)$, and the maximum of fitness $f\left(\mathbf{Q}_{(\xi, \theta, \beta)_{m}}\right)$ was set as the initial global optimal solution $g_{0}$. If the global optimal solution $g_{0}$ equaled $\mathbf{M}$, the algorithm broke and the current particle position was the optimal placement of the sensors, or else, Step 2 followed.

Step 2. (updating the PSO algorithm). The local optimal solution $g_{l}$ was determined by the fitness maximization of 10 particles when the velocity and position of every particle were updated. If $g_{l}>g_{0}, g_{0}$ is replaced with $g_{l}$ and the corresponding particle position was the current optimal placement for strain sensors; else, $g_{0}$ and placements of strain sensors were invariant and let $k=k+1$, moving to Step 3 .

Step 3. (terminating the PSO algorithm). The iteration did not break until the iteration number $k=k_{\max }$. If the current local optimal solution $g_{l}$ exceeded the previous global optimal solution $g_{0}$, the current particle position would be the optimal placement of strain sensors; else, the particle position that caused the previous $g_{0}$ would be the optimal solution for the optimization model of (14).

\section{Simulation and Experimental Results}

In order to assess the accuracy and effectiveness of the optimal sensor placement scheme, a simple cantilevered beam and a wing-like framework were subjected to different end-node static forces and the corresponding deformations were computed through iFEM. The cantilevered beam was a finite element model, and the framework was a physical model. Both structures were made of an aluminum alloy of $E=73000 \mathrm{Mpa}$ in Young's modulus, a Poisson ratio of $v=0.3$, and a density of $\rho=2712.63 \mathrm{~kg} / \mathrm{m}^{3}$. The framework was composed of three solid circular cross-section beams and middle plates. The span of each beam was $L=640 \mathrm{~mm}$ and of $R=10 \mathrm{~mm}$ in radius (Figure 3 ).

4.1. Simulation Tests. Firstly, the three different sensor placements were compared, two of which (C1 and $\mathrm{C} 2$ ) originated from the literature [16], while the third (C3) was the proposed optimal placement (Table 1). In lieu of the 


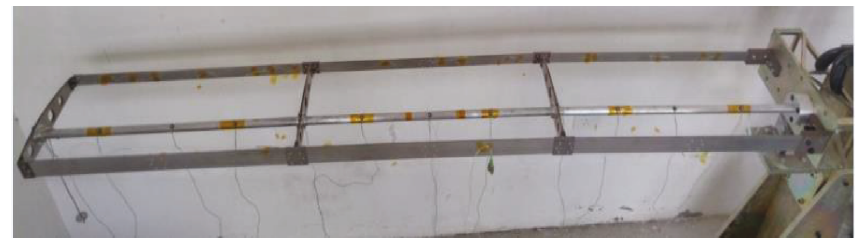

FIGURE 3: Wing-like framework.

TABLE 1: Schemes of sensor placement. Each location was written as $\left(\xi_{i}, \theta_{i}, \beta_{i}\right), i=1, \ldots, 6$.

\begin{tabular}{lccc}
\hline & $\mathrm{C} 1$ & $\mathrm{C} 2$ & $\mathrm{C} 3$ \\
\hline$\varepsilon_{1}$ & $\left(0,-120^{\circ}, 0^{\circ}\right)$ & $\left(-0.33,-120^{\circ}, 0^{\circ}\right)$ & $\left(-0.8,-90^{\circ}, 0^{\circ}\right)$ \\
$\varepsilon_{2}$ & $\left(0,-120^{\circ}, 45^{\circ}\right)$ & $\left(-0.33,120^{\circ}, 0^{\circ}\right)$ & $\left(-0.68,0^{\circ}, 0^{\circ}\right)$ \\
$\varepsilon_{3}$ & $\left(0,0^{\circ}, 0^{\circ}\right)$ & $\left(-0.33,0^{\circ}, 0^{\circ}\right)$ & $\left(-0.68,180^{\circ}, 45^{\circ}\right)$ \\
$\varepsilon_{4}$ & $\left(0,0^{\circ}, 45^{\circ}\right)$ & $\left(0.33,0^{\circ}, 45^{\circ}\right)$ & $\left(0.78,90^{\circ}, 0^{\circ}\right)$ \\
$\varepsilon_{5}$ & $\left(0,120^{\circ}, 0^{\circ}\right)$ & $\left(0.33,-120^{\circ}, 0^{\circ}\right)$ & $\left(0.8,180^{\circ}, 0^{\circ}\right)$ \\
$\varepsilon_{6}$ & $\left(0,120^{\circ}, 45\right)$ & $\left(0.33,120^{\circ}, 0^{\circ}\right)$ & $\left(0.8,0^{\circ}, 0^{\circ}\right)$ \\
$f\left(\mathbf{Q}_{(\xi, \theta, \beta)}\right)$ & 0.3286 & 2.6240 & 45.5918 \\
\hline
\end{tabular}

experimentally measured surface strains on the cantilevered beam, high-fidelity direct finite element analyses (ANSYS 14.5) were carried out, whereas for the beam member based on Timoshenko theory accurate modeling, a beam of 188 elements was employed. The model was divided into 200 elements, and the cross-section of the beam was divided into 120 sectors (Figure 4).

The free end of the beam model was statically loaded through a combination of two forces (Figure 5). The comparisons of iFEM deformation reconstructions of the cantilevered beam with three different schemes of placement are presented in Table 2.

In this paper, two evaluation criteria were defined as

$$
\begin{aligned}
\text { MER } & =\max \left|\operatorname{disp}^{\operatorname{ANSYS}}\left(x_{i}\right)-\operatorname{disp}{ }^{\text {iFEM }}\left(x_{i}\right)\right|, \\
\mathrm{RMS} & =\sqrt[2]{\sum_{i=1}^{201} \frac{\left(\operatorname{disp}^{\mathrm{ANSY}}\left(x_{i}\right)-\operatorname{disp}^{\mathrm{iFEM}}\left(x_{i}\right)\right)^{2}}{201}}, \\
i & =1, \ldots, 201,
\end{aligned}
$$

where $\operatorname{disp}\left(x_{i}\right)$ is the displacement of one node along the beam centroidal axis in one direction, the superscript "ANSYS" refers to the deformation value from ANSYS simulation, and "iFEM" refers to the predicted value computed from iFEM; MER and RMS indicate the maximum error and root-mean-square error of deformation reconstruction, respectively. Table 2 presents that the deformations reconstructed through iFEM were nearly similar to the ANSYS simulation.

In view of the fact that the errors of strain measurements consisted of the errors of strain sensor placement and the system error of the strain measuring device, which might impact the accuracy of the deformation reconstruction computed from (5), it was assumed that the errors of the sensor placement were set as follows:

$$
\begin{aligned}
\Delta \xi_{i} & \in[-0.05,0.05], \\
\Delta \theta_{i} & \in\left[-9^{\circ}, 10^{\circ}\right], \\
\Delta \beta_{i} & \in\left[-10^{\circ}, 10^{\circ}\right], \\
i & =1,2, \ldots, 6 .
\end{aligned}
$$

The system error of the strain measuring device was assumed to obey a Gaussian error distribution, which had zero mean value and three-standard deviations equal to $5 \%$ of the ANSYS simulation strain value, such as $\varepsilon^{\prime}=\varepsilon+\Delta \varepsilon$, $5 \% \varepsilon \leq \Delta \varepsilon \leq 5 \% \varepsilon$. These disturbances were added 1000 times, and the highest error was selected for the reconstruction accuracy. The comparison of deformation reconstructions with noise for three displacements is presented in Table 3.

As it was assumed, the computing might be abnormal when the difference among the eigenvalues of coefficient matrix $\mathbf{Q}$ in (12) was low. The lowest difference among the eigenvalues of coefficient matrix $\mathbf{Q}$ for sensor placement $\mathrm{C} 1$ was 0.3286 . The reconstruction accuracies in directions $X, Y$, and $Z$ dropped to $16.77 \mathrm{~mm}, 8.63 E 3 \mathrm{~mm}$, and $1.74 E 3 \mathrm{~mm}$ in the case of maximum error; the reconstruction accuracies were $9.69 \mathrm{~mm}, 5.23 E 3 \mathrm{~mm}$, and $1.19 E 3 \mathrm{~mm}$ in the case of root-mean-square error. The lowest difference among the eigenvalues of coefficient matrix $\mathbf{Q}$ for optimal sensor placement C3 was 45.5918 and the corresponding reconstruction accuracies in directions $X, Y$, and $Z$ only dropped to $0.026 \mathrm{~mm}, 1.39 \mathrm{~mm}$, and $0.79 \mathrm{~mm}$ in the case of maximum error; these were $0.015 \mathrm{~mm}, 0.53 \mathrm{~mm}$, and $0.41 \mathrm{~mm}$ in the case of root-mean-square error (Table 3). The stability of C3 exceeded the C1 stability.

4.2. Physical Model Tests. Following, the optimal sensor placement $\mathrm{C} 3$ and the normal placement $\mathrm{C} 1$ were used for the deformation reconstruction of the wing-like structure under the static end-node loadings (Figure 6(a)). The total weight of the load was $8.92 \mathrm{~kg}$ (Table 4).

The surface measured strains were captured by fiber Bragg grating (FBG) strain sensors. Also, the experimental shapes were captured through 3D optical measurement instruction (NDI Optotrak Certus, Figure 6(b)), which determined the position of the identification point with three CCD cameras to capture the infrared lights emitted by the position sensors (Figure 6(c)). Also, the number of position sensors used in this test was 18. The instruction was also used to 

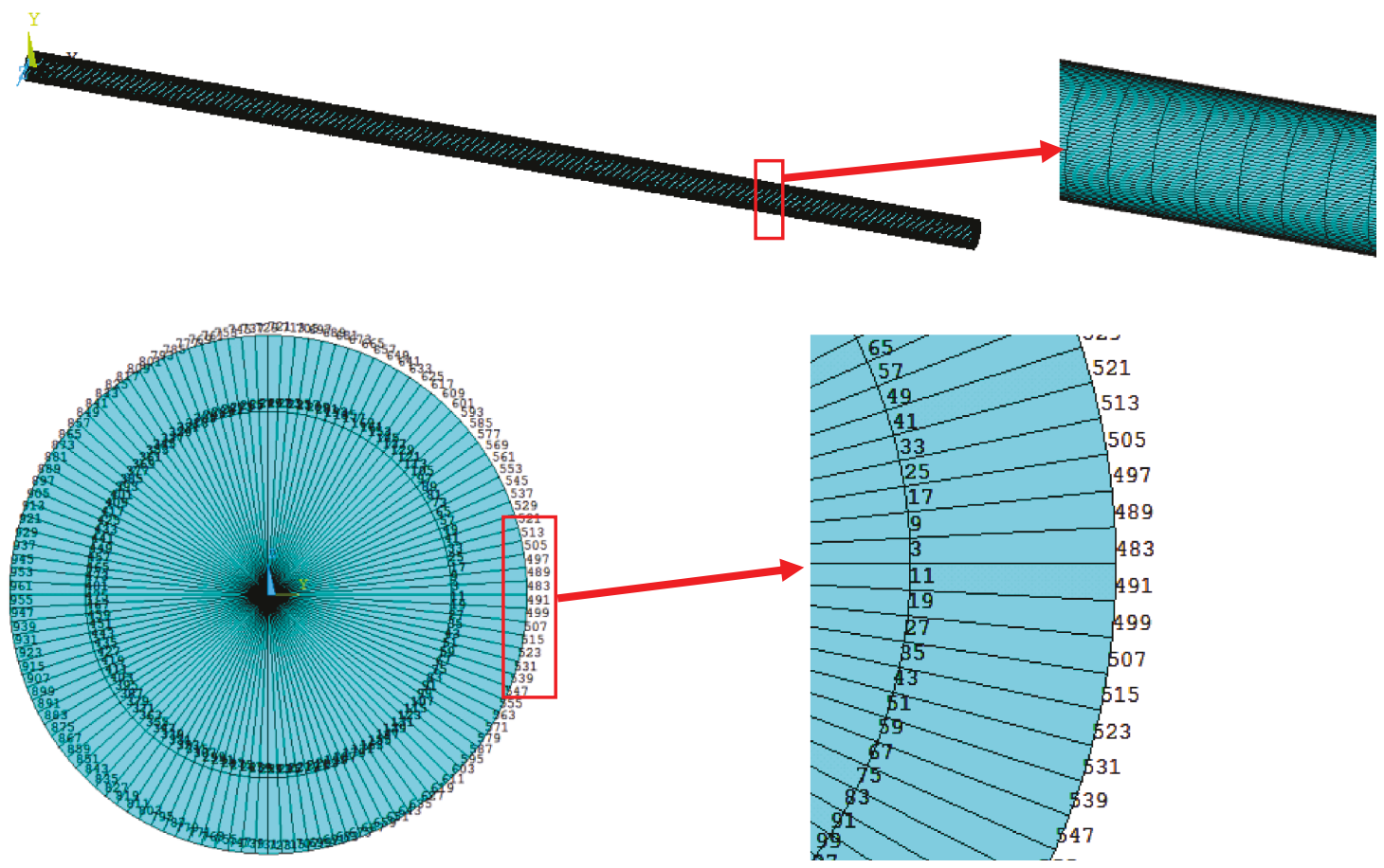

FIGURE 4: Finite element model of beam and the section division.

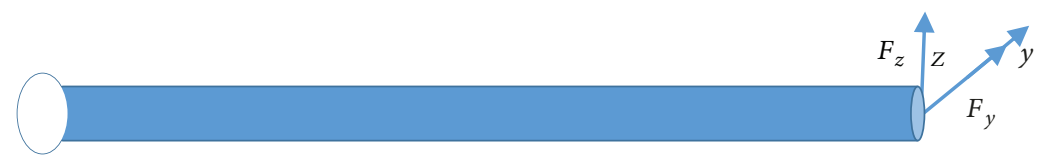

FIGURE 5: Free end loads: $F_{y}=-100 \mathrm{~N}, F_{\mathrm{z}}=150 \mathrm{~N}$.

TABLE 2: Comparison of reconstruction accuracies under three placements; displacements expressed in mm.

\begin{tabular}{lccc}
\hline & Max deformation in direction, $X$ & Max deformation in direction, $Y$ & Max deformation in direction, $Z$ \\
ANSYS & 0 & -16.28 & 24.42 \\
C1 & $4.4 E-6$ & -16.36 & 24.55 \\
C2 & $4.4 E-6$ & -16.22 & 24.33 \\
C3 & $1.13 E-16$ & -16.19 & 24.27 \\
\hline & MER in direction, $X$ & MER in direction, $Y$ & MER in direction, $Z$ \\
C1 & $4.4 E-06$ & 0.09 & 0.13 \\
C2 & $4.40 E-06$ & 0.09 & 0.15 \\
C3 & $1.13 E-16$ & 0.06 & 0.09 \\
\hline & RMS in direction, $X$ & 0.064 & RMS in direction, $Z$ \\
C1 & $2.54 E-06$ & 0.03 & 0.096 \\
C2 & $2.54 E-06$ & 0.02 & 0.06 \\
C3 & $6.54 E-17$ & RM in direction, $Y$ & 0.03 \\
\hline
\end{tabular}

assess the deformation computed from iFEM, where the accuracy of the NDI was $0.1 \mathrm{~mm}$. The FBG strain sensors were placed on the surfaces of three beams with the identical distributions of $\mathrm{C} 1$ and $\mathrm{C} 3$. As a FBG sensor could not overlap another sensor, it was difficult to place the two sensors on one point, such as at $\left(0,0^{\circ}, 0^{\circ}\right)$ and $\left(0,0^{\circ}, 45^{\circ}\right)$. Therefore, the placement scheme $\mathrm{C} 1$ was slightly changed (Table 5$)$. The comparison between the measured deformation captured from NDI and the reconstructed deformation computed from iFEM is presented in Table 6.

In Table $6, \mathrm{MD}^{\mathrm{NDI}}$ indicates the maximum deformation measured by/through NDI. MD ${ }^{\text {iFEMC1 }}$ and $\mathrm{MD}^{\mathrm{iFEMC3}}$ indicate the maximum deformation computed through iFEM with the sensor placement schemes of $\mathrm{C} 1$ and $\mathrm{C} 3 . \mathrm{MER}_{\mathrm{C} 1}$ 
TABLE 3: Comparisons among deformations computed from iFEM and deformations extracted from ANSYS. Computed results contained system errors, combining errors of sensor-locations with errors of strain-measurements. Displacements expressed in mm.

\begin{tabular}{lccc}
\hline & Max deformation in direction, $X$ & Max-deformation in direction, $Y$ & Max-deformation in direction, $Z$ \\
ANSYS & 0 & -16.28 & 24.42 \\
C1 & -16.77 & $8.61 E 3$ & $1.76 E 3$ \\
C2 & 0.065 & -12.75 & 21.84 \\
C3 & -0.026 & -14.89 & 25.21 \\
\hline & MER in direction, $X$ & MER in direction, $Y$ & MER in direction, $Z$ \\
C1 & 16.77 & $8.63 E 3$ & $1.74 E 3$ \\
C2 & 0.065 & 3.53 & 2.58 \\
C3 & 0.026 & 1.39 & 0.79 \\
\hline & RMS in direction, $Y$ & RMS in direction, $Z$ \\
C1 & RMS in direction, $X$ & $5.23 E 3$ & $1.19 E 3$ \\
C2 & 9.69 & 1.71 & 1.37 \\
C3 & 0.038 & 0.53 & 0.41 \\
\hline
\end{tabular}

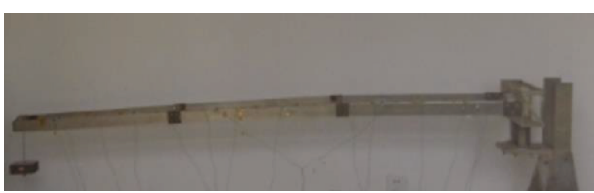

(a)

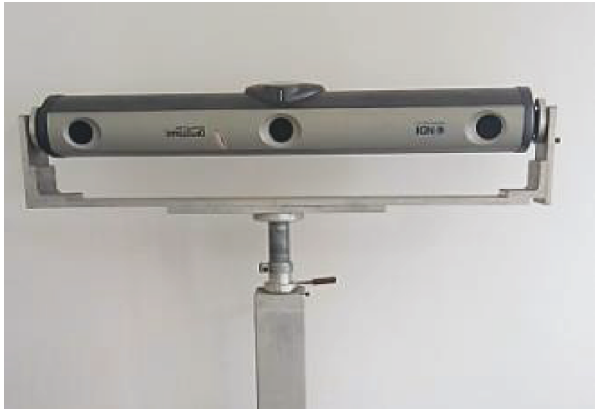

(b)

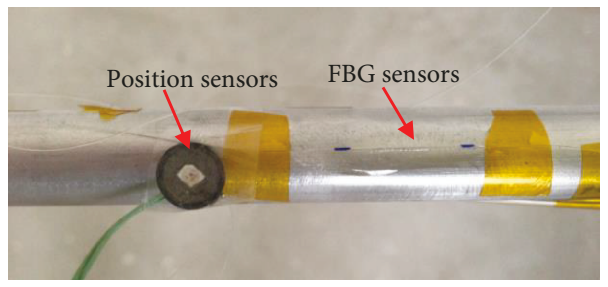

(c)

Figure 6: (a) Loading on end-node of entire structure. (b) NDI Optotrak Certus. (c) Position sensors and FBG sensors.

TABLE 4: Static loading cases.

\begin{tabular}{ccccccc}
\hline & 1 & 2 & 3 & 4 & 5 & 6 \\
\hline Loading & $0.94 \mathrm{~kg}$ & $2.73 \mathrm{~kg}$ & $3.72 \mathrm{~kg}$ & $6.14 \mathrm{~kg}$ & $7.25 \mathrm{~kg}$ & $8.92 \mathrm{~kg}$ \\
\hline
\end{tabular}

$\left(\mathrm{MER}_{\mathrm{C} 3}\right)$ and $\mathrm{RMS}_{\mathrm{C} 1}\left(\mathrm{RMS}_{\mathrm{C} 3}\right)$ were computed through (15). As the number of nodes of structural deformation measured through NDI with position was 18 , the number of the nodes (201) in (15) should be replaced by 18, while the measured values $\operatorname{disp}^{\text {ANSYS }}\left(x_{i}\right)$ were replaced by $\operatorname{disp}^{\mathrm{NDI}}\left(x_{i}\right)$, as captured from NDI. Moreover, the maximum relative error (MRE) was used to assess two schemes.

$$
\operatorname{MRE}=\frac{\mathrm{MER}}{\left|\mathrm{MD}^{\mathrm{NDI}}\right|} \%
$$

The results in Table 6 demonstrated that, with the C3 sensor placement, the iFEM could reconstruct the framework deformation with high precision, whereas in most loading cases (loading cases of 3 6), the results were more stable and precise than the results computed through iFEM with the $\mathrm{C} 1$ sensor placement (with placement $\mathrm{C} 3$, the relative errors were approximately 3\%; the highest error that occurred in loading case 6 was $6.1 \%$; while with placement $\mathrm{C} 1$, the relative errors increased from $3 \%$ to $11.4 \%$ as the load increased). In loading cases 1 and 2, the differences among iFEM computing and NDI measurements were higher than those in the other cases (in cases 1 and 2, the highest errors appeared at direction $Y$, such as the loading direction; the highest errors were $2.9 \mathrm{~mm}$ in case 1 and $3.4 \mathrm{~mm}$ in case 2, while the corresponding relative errors were $13.2 \%$ and $11.5 \%$, resp.), since a clearance existed between the first beam 
TABLE 5: C1changed scheme of sensor placement.

\begin{tabular}{ccccccc}
\hline & $\varepsilon_{1}$ & $\varepsilon_{2}$ & $\varepsilon_{3}$ & $\varepsilon_{4}$ & $\varepsilon_{5}$ & $\varepsilon_{6}$ \\
\hline $\mathrm{C} 1$ & $\left(0,-120^{\circ}, 0^{\circ}\right)$ & $\left(0.2,-120^{\circ}, 45^{\circ}\right)$ & $\left(0,0^{\circ}, 0^{\circ}\right)$ & $\left(0.2,0^{\circ}, 45^{\circ}\right)$ & $\left(0,120^{\circ}, 0^{\circ}\right)$ & $\left(0.2,120^{\circ}, 45^{\circ}\right)$ \\
\hline
\end{tabular}

TABLE 6: Comparison between NDI and iFEM reconstructions in loading case of end-node.

\begin{tabular}{|c|c|c|c|c|c|c|}
\hline & Direction $Y$ & Direction $Z$ & Direction $Y$ & Direction $Z$ & Direction $Y$ & Direction $Z$ \\
\hline & \multicolumn{2}{|c|}{1} & \multicolumn{2}{|c|}{2} & \multicolumn{2}{|c|}{3} \\
\hline $\mathrm{MD}^{\mathrm{NDI}}$ & $-21.9 \mathrm{~mm}$ & $0.2 \mathrm{~mm}$ & $-29.5 \mathrm{~mm}$ & $0.3 \mathrm{~mm}$ & $-36.6 \mathrm{~mm}$ & $0.7 \mathrm{~mm}$ \\
\hline $\mathrm{MD}^{\mathrm{iFEMC1}}$ & $-24.8 \mathrm{~mm}$ & $0.4 \mathrm{~mm}$ & $-27.4 \mathrm{~mm}$ & $0.4 \mathrm{~mm}$ & $-37.5 \mathrm{~mm}$ & $0.9 \mathrm{~mm}$ \\
\hline $\mathrm{MD}^{\mathrm{iFEMC} 3}$ & $-23.7 \mathrm{~mm}$ & $0.4 \mathrm{~mm}$ & $-26.1 \mathrm{~mm}$ & $0.4 \mathrm{~mm}$ & $-35.7 \mathrm{~mm}$ & $0.8 \mathrm{~mm}$ \\
\hline $\mathrm{MER}_{\mathrm{C} 1}$ & $2.9 \mathrm{~mm}$ & $0.3 \mathrm{~mm}$ & $2.1 \mathrm{~mm}$ & $0.2 \mathrm{~mm}$ & $1.1 \mathrm{~mm}$ & $0.3 \mathrm{~mm}$ \\
\hline $\mathrm{MER}_{\mathrm{C} 3}$ & $1.8 \mathrm{~mm}$ & $0.3 \mathrm{~mm}$ & $3.4 \mathrm{~mm}$ & $0.2 \mathrm{~mm}$ & $1.1 \mathrm{~mm}$ & $0.2 \mathrm{~mm}$ \\
\hline $\mathrm{RMS}_{\mathrm{C} 1}$ & $1.42 \mathrm{~mm}$ & $0.18 \mathrm{~mm}$ & $1.03 \mathrm{~mm}$ & $0.13 \mathrm{~mm}$ & $0.67 \mathrm{~mm}$ & $0.16 \mathrm{~mm}$ \\
\hline $\mathrm{RMS}_{\mathrm{C} 3}$ & $0.89 \mathrm{~mm}$ & $0.16 \mathrm{~mm}$ & $1.60 \mathrm{~mm}$ & $0.12 \mathrm{~mm}$ & $0.65 \mathrm{~m}$ & $0.12 \mathrm{~mm}$ \\
\hline $\mathrm{MRE}_{\mathrm{C} 1}$ & $13.2 \%$ & $150 \%$ & $7.1 \%$ & $66.7 \%$ & $3 \%$ & $42.9 \%$ \\
\hline \multirow{2}{*}{$\mathrm{MRE}_{\mathrm{C} 3}$} & $8.2 \%$ & $150 \%$ & $11.5 \%$ & $66.7 \%$ & $3 \%$ & $28.6 \%$ \\
\hline & \multicolumn{2}{|c|}{4} & \multicolumn{2}{|c|}{5} & \multicolumn{2}{|c|}{6} \\
\hline $\mathrm{MD}^{\mathrm{NDI}}$ & $-59.6 \mathrm{~mm}$ & $0.7 \mathrm{~mm}$ & $-67.3 \mathrm{~mm}$ & $1.1 \mathrm{~mm}$ & $-75.3 \mathrm{~mm}$ & $1.1 \mathrm{~mm}$ \\
\hline $\mathrm{MD}^{\mathrm{iFEMC1}}$ & $-62.2 \mathrm{~mm}$ & $0.9 \mathrm{~mm}$ & $-73.1 \mathrm{~mm}$ & $1.3 \mathrm{~mm}$ & $-83.8 \mathrm{~mm}$ & $1.4 \mathrm{~mm}$ \\
\hline $\mathrm{MD}^{\mathrm{iFEMC} 3}$ & $-59.3 \mathrm{~mm}$ & $0.8 \mathrm{~mm}$ & $-69.6 \mathrm{~mm}$ & $1.2 \mathrm{~mm}$ & $-79.9 \mathrm{~mm}$ & $1.2 \mathrm{~mm}$ \\
\hline $\mathrm{MER}_{\mathrm{C} 1}$ & $2.6 \mathrm{~mm}$ & $0.3 \mathrm{~mm}$ & $5.8 \mathrm{~mm}$ & $0.3 \mathrm{~mm}$ & $8.6 \mathrm{~mm}$ & $0.4 \mathrm{~mm}$ \\
\hline $\mathrm{MER}_{\mathrm{C} 3}$ & $1.7 \mathrm{~mm}$ & $0.2 \mathrm{~mm}$ & $2.3 \mathrm{~mm}$ & $0.2 \mathrm{~mm}$ & $4.6 \mathrm{~mm}$ & $0.3 \mathrm{~mm}$ \\
\hline $\mathrm{RMS}_{\mathrm{C} 1}$ & $1.49 \mathrm{~mm}$ & $0.16 \mathrm{~mm}$ & $2.86 \mathrm{~mm}$ & $0.18 \mathrm{~mm}$ & $4.16 \mathrm{~mm}$ & $0.21 \mathrm{~mm}$ \\
\hline $\mathrm{RMS}_{\mathrm{C} 3}$ & $0.84 \mathrm{~mm}$ & $0.13 \mathrm{~mm}$ & $1.43 \mathrm{~mm}$ & $0.15 \mathrm{~mm}$ & $2.4 \mathrm{~mm}$ & $0.15 \mathrm{~mm}$ \\
\hline $\mathrm{MRE}_{\mathrm{C} 1}$ & $4.4 \%$ & $42.9 \%$ & $8.6 \%$ & $27.3 \%$ & $11.4 \%$ & $36.4 \%$ \\
\hline $\mathrm{MRE}_{\mathrm{C} 3}$ & $2.9 \%$ & $28.6 \%$ & $3.4 \%$ & $18.2 \%$ & $6.1 \%$ & $27.3 \%$ \\
\hline
\end{tabular}

and the ring flange. The deformation on the clamped endnode should be zero in theory, but as a clearance existed, the boundary condition in iFEM changed. Therefore, the accuracy and stability of deformation reconstruction of the entire structure changed slightly. As the load increased, the clearance disappeared, whereas the reconstruction became accurate and steady.

\section{Conclusions}

This paper was focused on the discovery of a feasible standard to optimize the placement of the strain sensors in iFEM. The iFEM could be used to reconstruct the structural deformation of the beam only with in situ surface strain measurement data and a low number of structure parameters, such as the length and radius of beam, as well as the Poisson ratio. Adversely, through the current study, it was discovered that the locations where the strain sensors were placed would impact the accuracy and stability of deformation reconstruction. Consequently, a minimum change among the matrix eigenvalues was proposed to determine the eigenvalue distribution of the coefficient matrix (coefficient matrix was associated to the relationship among section strains and surface strain measurement data), to select the optimal placement of sensors to maintain the accuracy and stability of the deformation reconstruction with iFEM. The simulation and physical model tests demonstrated that the C3 optimal placement scheme had good potential in maintaining the structural deformation reconstruction with iFEM. Nevertheless, low fluctuations existed in the physical model tests, as clearance existed between the first beam and the ring flange. Moreover, errors occurred in the process of pasting sensors, which would also impact the reconstruction results.

\section{Data Availability}

The data used to support the findings of this study are available from the corresponding author upon request.

\section{Conflicts of Interest}

The authors declare that there is no conflict of interest regarding the publication of this paper. 


\section{Acknowledgments}

This work was financially supported by the National Natural Science Foundation of China through Grants 51775401 and 51490660, the National Key Basic Research Program of China through Grant 2015CB857100, the CAS "Light of West China” Program no. 2016-QNXZ-A-7, and the joint research fund in astronomy (no. U1731135) under a cooperative between the National Science Foundation of China (NSFC) and Chinese Academy of Sciences (CAS). Special appreciation was given to the editor and the anonymous reviewers for their careful reviews and valuable comments.

\section{References}

[1] I. D. M. Esper and P. E. E. Rosa, "Heading controller for a fixed wing UAV with reduced control surfaces based on ANFIS," in 2015 IEEE International Conference on Dependable Systems and Networks Workshops, pp. 118-123, Rio de Janeiro, Brazil, June 2015.

[2] C.-g. Pak, "Wing shape sensing from measured strain," AIAA Journal, vol. 54, no. 3, pp. 1068-1077, 2016.

[3] T. Pisanu, F. Buffa, M. Morsiani, P. Claudio, and P. Sergio, "Thermal behavior of the Medicina 32-meter radio telescope," in Proceedings of SPIE 7739, Modern Technologies in Spaceand Ground-based Telescopes and Instrumentation, vol. 773935, San Diego, CA, USA, July 2010.

[4] Z. Yong, B. Hong, D. Xuechao, and F. Hongmei, "Research of situ strain of measuring flexible truss deformation based on fuzzy network method," in Proceedings of the 2016 International Conference on Computer Engineering, Information Science \& Application Technology (ICCIA 2016), pp. 401-410, September 2016.

[5] D. Ganotra, "Object reconstruction in multilayer neural network based profilometry using grating structure comprising two regions with different spatial periods," Optics and Lasers in Engineering, vol. 42, no. 2, pp. 179-192, 2004.

[6] S. Rapp, L. H. Kang, J. H. Han, U. C. Mueller, and H. Baier, "Displacement field estimation for a two-dimensional structure using fiber Bragg grating sensors," Smart Materials and Structures, vol. 18, article 025006, 2009.

[7] H. I. Kim, L. H. Kang, and J. H. Han, "Shape estimation with distributed fiber Bragg grating sensors for rotating structures," Smart Materials and Structures, vol. 20, no. 3, article 035011, 2011.

[8] P. B. Bogert, E. D. Haugse, and R. E. Gehrki, "Structural shape identification from experimental strains using a modal transformation technique," in 44th AIAA/ASME/ASCE/AHS/ASC Structures, Structural Dynamics, and Materials Conference, Norfolk, Virginia, April 2003.

[9] R. Glaser, V. Caccese, and M. Shahinpoor, "Shape monitoring of a beam structure from measured strain or curvature," Experimental Mechanics, vol. 52, no. 6, pp. 591-606, 2012.

[10] C. Jute, W. Ko, and C. Stephens, "Deformed shape calculation of a full-scale wing using fiber optic strain data from a ground loads," Rept. TP-215975, TEST NASA Langley Research Center, Hampton, VA, USA, 2011.

[11] S. Shkarayev, R. Krashantisa, and A. Tessler, "An inverse interpolation method utilizing in-flight strain measurements for determining loads and structural response of aerospace vehicles," in Proceedings of 3rd International Workshop on Structural Health Monitoring, Stanford, CA, USA, 2001.

[12] S. Shkarayev, A. Raman, and A. Tessler, "Computational and experimental validation enabling a viable in-flight structural health monitoring technology," in Proceedings of the First European Workshop Structural Health Monitoring 2002, Cachan, Paris, France, July 2002.

[13] A. Tessler and J. L. Spangler, "Inverse FEM for full-field reconstruction of elastic deformations in shear deformable plates and shells," in 2nd European Workshop on Structural Health Monitoring, p. 9, Munich, Germany, July 2004.

[14] A. Tessler and J. L. Spangler, "A least-squares variational method for full-field reconstruction of elastic deformations in shear-deformable plates and shells," Computer Methods in Applied Mechanics and Engineering, vol. 194, no. 2-5, pp. 327-339, 2005.

[15] M. Gherlone, P. Cerracchio, M. Mattone, M. Di Sciuva, and A. Tessler, "Shape sensing of 3D frame structures using an inverse finite element method," International Journal of Solids and Structures, vol. 49, no. 22, pp. 3100-3112, 2012.

[16] M. Gherlone, P. Cerracchio, M. Mattone, M. di Sciuva, and A. Tessler, "An inverse finite element method for beam shape sensing: theoretical framework and experimental validation," Smart Materials and Structures, vol. 23, no. 4, article 45027, 2014.

[17] P. Costas and L. Geert, "The effect of prediction error correlation on optimal sensor placement in structural dynamics," Mechanical Systems and Signal Processing, vol. 28, pp. 105127, 2012.

[18] D. S. Li, H. N. Li, and C. P. Fritzen, "Load dependent sensor placement method: theory and experimental validation," Mechanical Systems and Signal Processing, vol. 31, pp. 217227, 2012.

[19] Z. Yong, B. Hong, D. Xuechao, and F. Hongmei, “The application research of inverse finite element method for frame deformation estimating," International Journal of Aerospace Engineering, vol. 2017, Article ID 1326309, 8 pages, 2017.

[20] H. Kunsoo and J. L. Stein, "Well-conditioned observer design for observer-based monitoring systems," Journal of Dynamic Systems, Measurement and Control, vol. 117, no. 4, pp. 592599, 1995. 


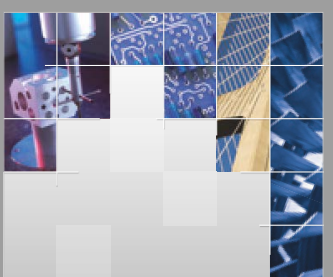

\section{Enfincering}
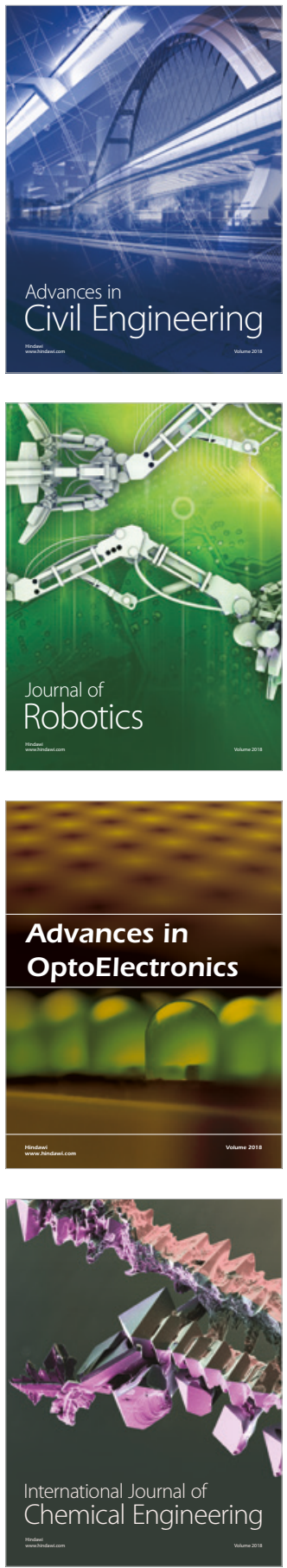

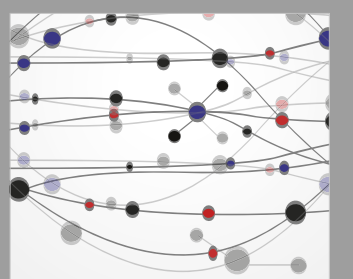

\section{Rotating \\ Machinery}

The Scientific World Journal

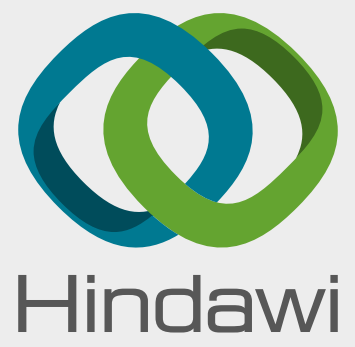

Submit your manuscripts at

www.hindawi.com
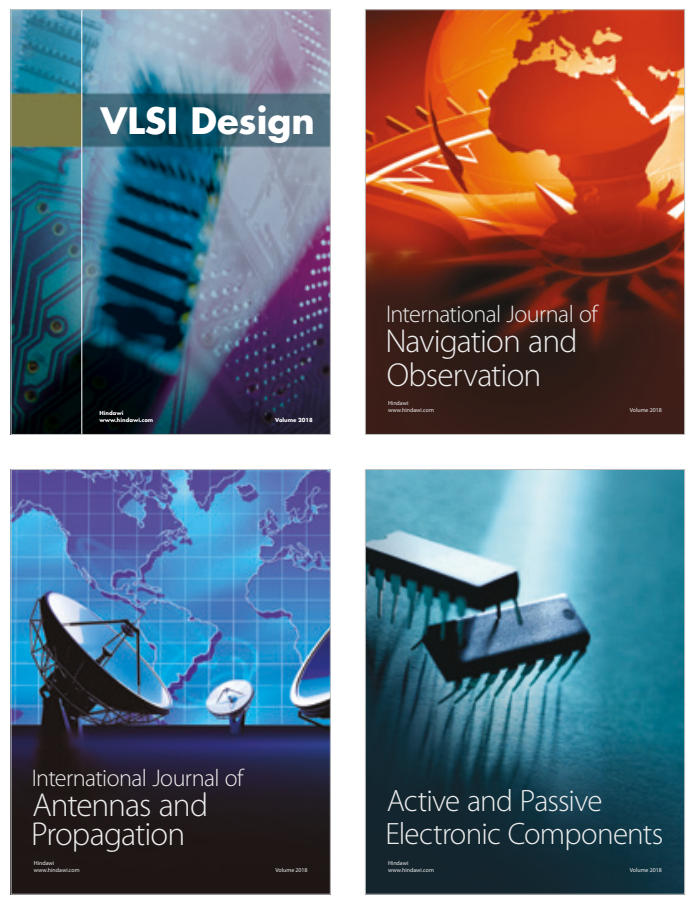
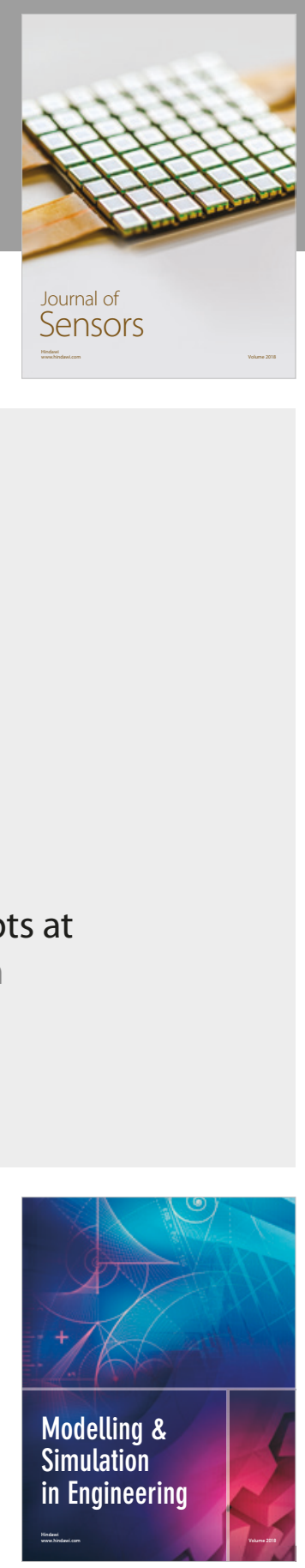

\section{Advances \\ Multimedia}
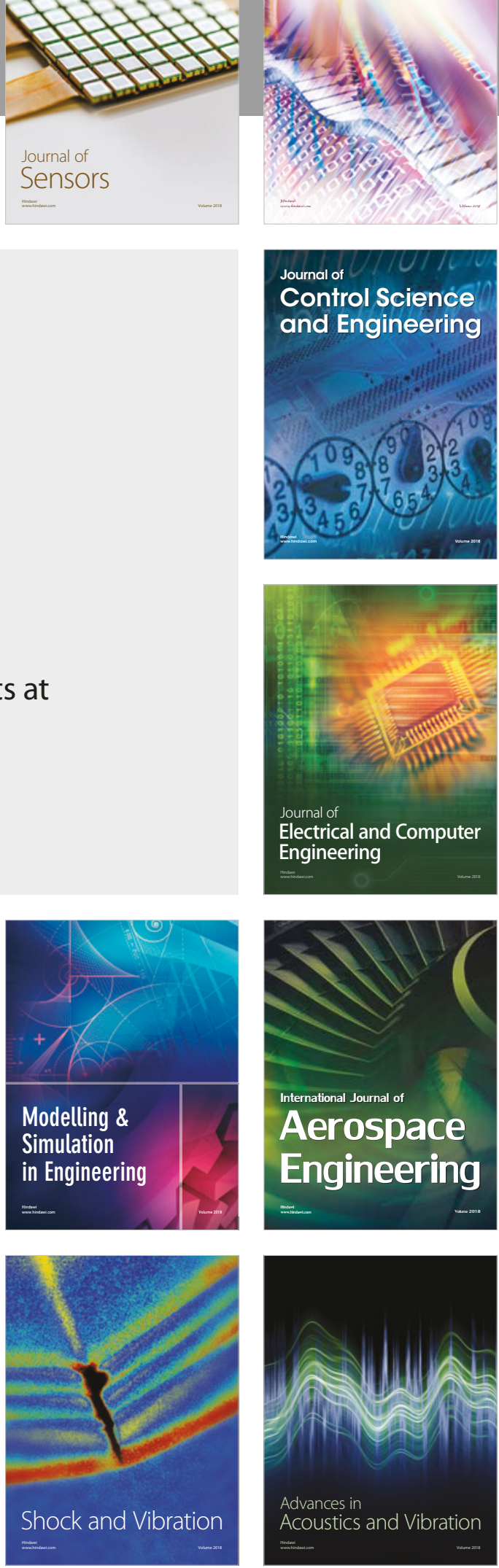